\title{
Clinical and radiological association with positive lumbar discography in patients with chronic low back pain
}

Authors William Omar Contreras López¹, Emiliano Neves Vialle², Carolina Casas Anillo², Mauricio Guzmao², Luiz Roberto Gomes Vialle ${ }^{2}$

Institutions $\quad{ }^{1}$ Functional Neurosurgery Department, Universitäts Klinikum, Freiburg, Germany.

${ }^{2}$ Spine Surgery Department, Hospital Universitario de Cajuru, Catholic University of Paraná, Brazil

${ }^{3}$ Rosario University, Bogota, Colombia

\begin{tabular}{|c|c|}
\hline $\begin{array}{l}\text { Assessment of CoE for } \\
\text { individual studies of } \\
\text { diagnostic test } \\
\text { evaluation* }\end{array}$ & \\
\hline \multicolumn{2}{|l|}{ Study design } \\
\hline Prospective cohort design & \\
\hline Retrospective cohort design & - \\
\hline Case-control design & \\
\hline \multicolumn{2}{|l|}{ Methods } \\
\hline $\begin{array}{l}\text { Broad spectrum of patients } \\
\text { with expected condition }\end{array}$ & - \\
\hline $\begin{array}{l}\text { Appropriate reference } \\
\text { standard used }\end{array}$ & - \\
\hline $\begin{array}{l}\text { Adequate description of test } \\
\text { and reference for replication }\end{array}$ & • \\
\hline $\begin{array}{l}\text { Blinded comparison with } \\
\text { appropriate reference }\end{array}$ & \\
\hline $\begin{array}{l}\text { Reference standard performed } \\
\text { independently of test }\end{array}$ & \\
\hline Overall class of evidence & IV \\
\hline \multicolumn{2}{|c|}{$\begin{array}{l}{ }^{*} \text { Blank box indicates criterion not } \\
\text { met, could not be determined, or } \\
\text { information not reported by author } \\
\text { or was not reported. }\end{array}$} \\
\hline $\begin{array}{l}\text { The definiton for classes of } \\
\text { evidence for diagnostic test } \\
\text { evaluations is available in the W } \\
\text { Appendix at www.aospine.org/E }\end{array}$ & $\begin{array}{l}\text { eb } \\
\text { ESSJ. }\end{array}$ \\
\hline
\end{tabular}

Study design: Retrospective cohort study.

Objectives: To find out (1) if magnetic resonance imaging (MRI) findings associated with positive discography in patients with lumbar discogenic pain are caused by degenerative disc disease (DDD). (2) If clinical risk factors associated with positive discography in patients with lumbar discogenic pain are caused by DDD.

Methods: Thirty-three discographies were performed in 20 consecutive patients with chronic low back pain (LBP). All examinations were performed in the lumbar spine between L3 and S1. Patient assessment consisted of a clinical and radiological examination through a protocol that contained data on the history, visual analogue scale for pain (VAS), functional questionnaire (Oswestry), and MRI findings. Discography was considered positive using the Walsh's criteria. We examined the association between MRI and clinical findings with positive discography using logistic regression.

Results: Fourteen discographies (42\%) were positive and 19 (58\%) were negative. The mean age of patients with positive discography was 40.7 years (range, 25-56 years) and negative discography 43.1 years (range, 30-55 years). Men had a positive discography rate of $43.5 \%$ and women $40 \%$. Patients with LBP had reduced odds of a positive discography compared with those with LBP and sciatica (OR=.5; 95\% CI: 0.1-2.7); however, this association was not statistically significant. Patients with more than four previous episodes of pain versus patients with one to four episodes had greater odds of a positive discography (OR=3.8; 95\% CI: 0.07-184); but this association was not statistically significant. Patients with various pathologies on MRI had greater odds of a positive discography; however, these associations were not statistically significant either.

Conclusions: Patients with a chief complaint of LBP associated with sciatica, with more than four episodes of previous LBP exacerbations and the presence of a high intensity zone (HIZ) on MRI have a higher rate of positive discography. These findings are not statistically significant, probably due to a small sample size. During discography, we found the end point resistance to be more prevalent in asymptomatic discs. 


\section{STUDY RATIONALE}

Discography is a puncture and contrast injection into the intervertebral disc. Its aim is to correlate clinical symptoms with imaging findings for the study of discs for which MRI findings are equivocal and confirming patients as discectomy candidates. A positive pain response involves pain consistent with the patient's symptoms, produced with low injection volume, which usually correlates with a visible disc rupture. Although, only disc rupture without concordant pain is considered a negative discography.

- Discography is usually performed as an additional confirmatory procedure after other imaging studies, such as X-rays and MRI, when doubtful if the origin of low back pain (LBP) is the intervertebral disc.

- This study evaluates the association between clinical risk factors and MRI findings with positive discography to identify patients for whom the diagnosis of LBP caused by discogenic disease could be achieved by less invasive procedures, such as clinical symptoms and MRI.

\section{OBJECTIVES}

To find out: (1) If MRI findings associated with positive discography in patients with lumbar discogenic pain are caused by degenerative disc disease (DDD)? (2) If clinical risk factors associated with positive discography in patients with lumbar discogenic pain are caused by DDD? (3) Another aim was to evaluate the resistance of the disc during the discography.

\section{METHODS}

Study design: Retrospective cohort study.

\section{Inclusion criteria:}

- Patients with chronic LBP who did not respond to conservative medical treatment including physical therapy for 6 months.

- Intolerance to prolonged standing and sitting.

- No motor weakness, corresponding to a nerve root.

- Normal reflexes.

- DDD, with a black disc (low intensity in T2) on MRI.

- Discography on record.

\section{Exclusion criteria:}

- Other nondegenerative diseases (abnormal bone structure with spondylolisthesis and/or sequelae of fractures)

- Thoracic or abdominal pain

- Motor weakness

- Pregnancy

- Coagulopathies

- Known allergy to any medications or dye

- Prior surgery for disc pathology

- Systemic infection or infection in puncture site

- Neurological disease

\section{Patient population and selection (Fig 1):}

- Twenty consecutive adult patients who had undergone an MRI and discography for the assessment of chronic LBP with suspected DDD were selected for analysis.

- Patients were selected from the records of a surgical consultation outpatient clinic at Cajuru University Hospital - PUC (Pontifical Catholic University) in Curitiba, Brazil, between December 2009 and March 2010.

\section{Procedure and imaging data for discography:}

- Patients had ECG monitoring of pulsoximetry and blood pressure during the procedure. Intravenous antibiotic was administered 30 minutes before the procedure. Discography was performed as an outpatient procedure [1].

- Patients were positioned prone on the image intensifier table, with lumbar asepsis, and placement of sterile drapes. The procedure required them to clearly understand questions that asked for the discography, and be able to respond clearly if the pain produced by the contrast medium intradiscal injection was exactly the same as their primary pain complaint (Fig 2). (More details of the procedure and evaluation is provided in the Web Appendix) 


\section{Discography assessment:}

The following measurements were made:

- Visual analogue scale (VAS) for pain during the examination

- Volume injected

- Disc resistance (resistance versus no resistance)

Positive discography was defined as a reproduction of the patient's pain when the contrast medium was injected.

\section{MRI assessment:}

The following types of disc degeneration on MRI and subsequent relationship with the results of the discography were assessed:

- Normal disc

- Just disc degeneration (black disc)

- Degeneration + disc protrusion

- Degeneration + disc extrusion

- Degeneration + HIZ + modic changes

(More details of the definition of HIZ and modic changes are provided in the Web Appendix, available at www. aospine.org/ebsj.)

\section{Clinical risk factor assessment:}

- Age

- Gender

- Primary pain complaint as either (1) LBP only or (2) back pain associated with sciatica.

- Number of previous episodes of pain exacerbations:

- None

- 1 to 4

- More than 4

- Baseline VAS pain score

- Baseline Oswestry score

\section{Analysis:}

- Descriptive analyses were performed using measures of central tendency and dispersion for continuous variables and proportions for categorical variables.

- To determine statistically significant differences in continuous variables the Student $t$-test or the nonparametric Wilcoxon rank sum (Mann-Whitney) were used when the assumption of normality was not fulfilled.

- To determine statistically significant differences in categorical variables, the chi-squared test or Fisher exact test was used when cell counts were less than 10.
- We calculated crude and adjusted odds ratios (ORs) and $95 \%$ confidence intervals (CI) examining the association between clinical factors and MRI findings with positive discography using logistic regression (Table 1).

- All tests were two-tailed for a type I error (alpha error) of 0.05 .

- Sensitivity and specificity were reported from the univariate logistic regression models.

Fig 1 Patient sampling and selection.

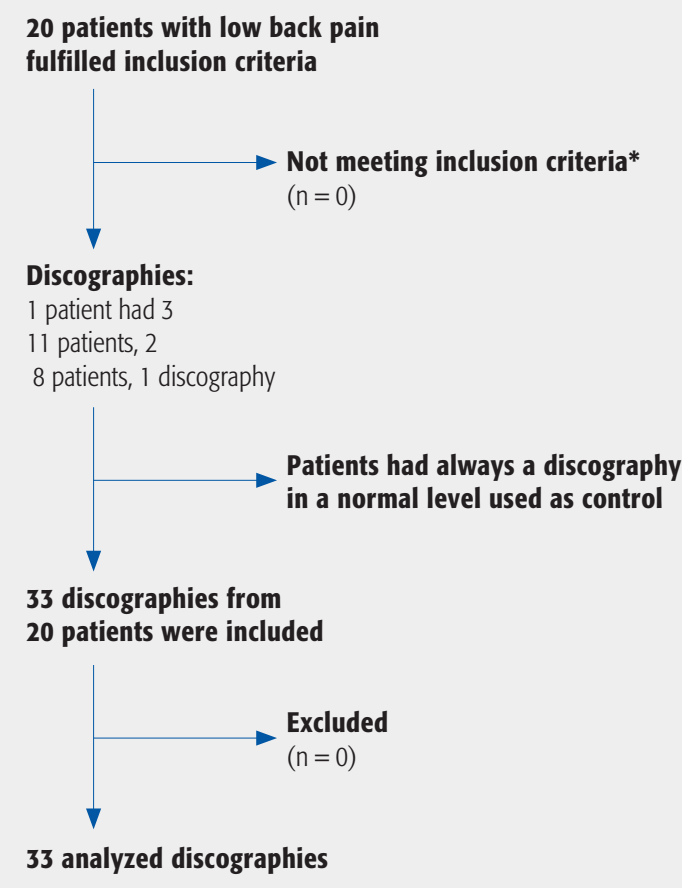


Table 1 Crude and adjusted odd ratios for the different variables of the study regarding positivity result of the test.

\begin{tabular}{|c|c|c|c|c|c|}
\hline & \multicolumn{2}{|c|}{ Discography result } & \multirow[t]{2}{*}{$P$} & \multirow[t]{2}{*}{ OR* (Cl 95\%) } & \multirow[t]{2}{*}{$\mathrm{OR}^{\dagger}(\mathrm{Cl}$ 95\%) } \\
\hline & N Positive & N Negative & & & \\
\hline Age, mean (SD) y & $41(9.3)$ & $45(7.9)$ & $.3^{\ddagger}$ & $0.9(0.9-1.0)$ & $0.9(0.8-1.0)$ \\
\hline Gender & & & $.7^{\S}$ & & \\
\hline Women & $5(50 \%)$ & $5(50 \%)$ & & 1 & 1 \\
\hline Men & $9(43 \%)$ & $12(57 \%)$ & & $0.7(0.1-3.4)$ & $1.7(0.2-12)$ \\
\hline Complaint & & & $.4^{\S}$ & & \\
\hline Lumbar pain and ciatica & $8(53 \%)$ & $7(47 \%)$ & & 1 & 1 \\
\hline Lumbar pain & $6(37 \%)$ & $10(62 \%)$ & & $0.4(0.1-2.2)$ & $0.5(0.1-2.7)$ \\
\hline Previous episodes & & & $.4^{\S}$ & & \\
\hline None & $1(50 \%)$ & $1(50 \%)$ & & 1 & 1 \\
\hline $1-4$ & $5(33 \%)$ & $10(66.7 \%)$ & & $0.5(0.02-9.76)$ & $0.73(0.01-33.1)$ \\
\hline$>4$ & $8(57 \%)$ & $6(43 \%)$ & & $1.3(0.06-26)$ & $3.8(0.07-184)$ \\
\hline Previous treatment & & & $.6^{\S}$ & & \\
\hline No & $2(33,33 \%)$ & $4(66,67 \%)$ & & 1 & 1 \\
\hline Yes & $12(48 \%)$ & $13(52 \%)$ & & $1.8(0.3-12)$ & $5.8(0.4-85)$ \\
\hline Previous surgery & & & $.3^{\S}$ & & \\
\hline No & $10(40 \%)$ & $15(60 \%)$ & & 1 & 1 \\
\hline Yes & $4(67 \%)$ & $2(33 \%)$ & & $2.9(0.5-19.5)$ & $3.3(0.4-25)$ \\
\hline $\begin{array}{l}\text { Visual analogue scale } \\
\text { lumbar, mean (SD) cm }\end{array}$ & $6.8(2)$ & $7.6(1.0)$ & $.4^{\S}$ & $0.6(0.4-1.1)$ & $0.6(0.3-1.1)$ \\
\hline $\begin{array}{l}\text { Visual analogue scale leg, } \\
\text { mean (SD), cm }\end{array}$ & $2.8(3.6)$ & $3.4(3.6)$ & $.4 \S$ & $0.9(0.8-1.1)$ & $0.9(0.8-1.2)$ \\
\hline Oswestry, mean (SD) & $36(11)$ & $44(12.3)$ & $.05^{\S}$ & $0.9(0.8-1.0)$ & $0.9(0.8-1.0)$ \\
\hline \multicolumn{6}{|l|}{ Findings on MRI } \\
\hline Black disc alone & $2(15 \%)$ & $11(85 \%)$ & $.009^{\S}$ & $0.1(0.015-0.5)$ & $0.1(0.019-1.07)$ \\
\hline Protrusion & $6(67 \%)$ & $3(33 \%)$ & $.2^{\S}$ & $3.5(0.7-18)$ & $3.9(0.6-24)$ \\
\hline Hernia & $3(75 \%)$ & $1(25 \%)$ & $.3^{\S}$ & $4.3(0.4-47)$ & $6.3(0.5-78)$ \\
\hline $\mathrm{Hiz}$ & $4(80 \%)$ & $1(20 \%)$ & $.1^{\S}$ & $6.5(0.62-65.7)$ & $8.5(0.8-91.5)$ \\
\hline Modic & $3(75 \%)$ & $1(25 \%)$ & $.3^{\S}$ & $4.4(0.4-47.6)$ & $6.4(0.5-73.3)$ \\
\hline
\end{tabular}

* Odds ratio (OR) crude. OR greater than one (1) are associated with positive discography (95\% confidence intervals $[\mathrm{CI}$ that include 1.0 represent non-statistically significant associations).

$\dagger$ OR adjusted. Adjusted for variables Hiz and modic.

‡ Wilcoxon-rank sum test (Mann-Whitney).

$\S \chi^{2}$-test.
Table 2 Characteristics of the base.*

\begin{tabular}{ll}
\hline Age, mean (SD) y & $43.2(8.64)$ \\
\hline Gender & \\
\hline Male & $10(32 \%)$ \\
\hline Female & $21(68 \%)$ \\
\hline Complaint & \\
\hline Low back pain & $16(52 \%)$ \\
\hline Low back pain and sciatica & $15(48 \%)$ \\
\hline Previous episodes & \\
\hline None & $2(6 \%)$ \\
\hline 1-4 & $15(48 \%)$ \\
\hline >4 & $14(45 \%)$ \\
\hline Previous treatments & \\
\hline Yes & $25(81 \%)$ \\
\hline No & $6(19 \%)$ \\
\hline Previous spinal surgery & \\
\hline Yes & $17(45 \%)$ \\
\hline No & $6(19 \%)$ \\
\hline VAS lumbar, mean (SD), cm & $7.26(1.6)$ \\
\hline VAS leg, mean (SD), cm & $3.16(3.6)$ \\
\hline Oswestry, mean (SD) & $40.64(12.1)$ \\
\hline Level & \\
\hline L2-L3 & $13(42 \%)$ \\
\hline L3-L4 & $9(29 \%)$ \\
\hline L4-L5 & $4(13 \%)$ \\
\hline L5-S1 & $6(19 \%)$ \\
\hline Findings on MRI & $13(42 \%)$ \\
\hline Only black disc & $11(35 \%)$ \\
\hline Protusion & \\
\hline Hernia & \\
\hline Hiz & \\
\hline Modic & \\
\hline Result of discography & \\
\hline Positive & \\
\hline Negative & \\
\hline
\end{tabular}

* VAS indicates visual analogue scale; MRI, magnetic resonance imaging. 
Table 3 Sensitivities and specificities of magnetic resonance imaging findings for the diagnosis of discogenic back pain.

\begin{tabular}{|c|c|c|c|c|}
\hline \multirow[b]{2}{*}{ Positive results } & \multicolumn{2}{|c|}{ Discography } & \multirow[t]{2}{*}{$\begin{array}{c}\text { Sensitivity \% } \\
(95 \% \mathrm{CI})\end{array}$} & \multirow[t]{2}{*}{$\begin{array}{c}\text { Specificity \% } \\
(95 \% \mathrm{CI})\end{array}$} \\
\hline & Positive & Negative & & \\
\hline $\begin{array}{l}\text { Black disc as } \\
\text { only finding }\end{array}$ & $2(15 \%)$ & $11(84 \%)$ & $14(1.78-42.8)$ & $35.29(14.2-61.6)$ \\
\hline Bulging & $6(67 \%)$ & $3(33 \%)$ & $43(17.6-71.1)$ & $82(56.5-96.2)$ \\
\hline Hernia & $3(75 \%)$ & $1(25 \%)$ & $21(4.6-50.8)$ & 94 (71.3-99.8) \\
\hline $\mathrm{HIZ}$ & $4(80 \%)$ & $1(20 \%)$ & $28(8.39-58.1)$ & 94 (71.3-99.8) \\
\hline Modic & $3(75 \%)$ & $1(25 \%)$ & $21.4(4.6-50.8)$ & $94(71.3-99.8)$ \\
\hline
\end{tabular}

Table 4 Relationship between characteristics of the discography with test results.*

\begin{tabular}{llll}
\hline $\begin{array}{l}\text { Characteristics of } \\
\text { discography }\end{array}$ & $\begin{array}{l}\text { Positive } \\
\text { discography }\end{array}$ & $\begin{array}{l}\text { Discography } \\
\text { negative }\end{array}$ & $P$ \\
\hline $\begin{array}{l}\text { VAS during the } \\
\text { examination }\end{array}$ & $8.92(8-10)$ & $0.74(2-0)$ & .003 \\
\hline Volume injected & $1.75 \mathrm{mLI}(13)$ & $2.18 \mathrm{~mL}(1.5-3)$ & $\mathrm{NS}$ \\
\hline Disc resistance & & & \\
\hline With resistance & $6(31.58 \%)$ & $13(68.42 \%)$ & .014 \\
\hline Without resistance & $7(50 \%)$ & $7(50 \%)$ & $\mathrm{NS}$ \\
\hline
\end{tabular}

* VAS indicates visual analogue scale; NS, no significance.

Fig 2 AP image intensifier showing the moment when the dye is injected into a L4-L5 disc discography.

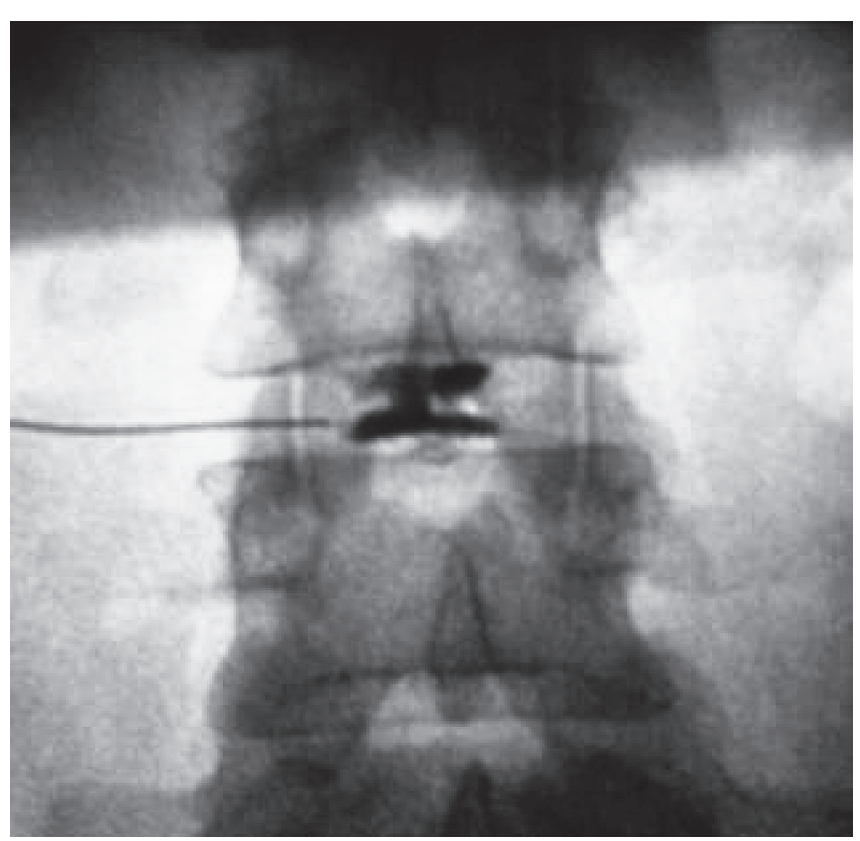

\section{RESULTS}

\section{General demographics}

- All discographies were performed on the lumbar spine between L3 and S1.

- A total of 33 discograms were performed in these 20 patients. One patient had 3 degenerate levels examined, 11 patients had 2 levels examined, and 8 had 1 level.

- Of 33 examinations performed, 14 were positive (42\%) and 19 negative $(58 \%)$. None of the normal discs on MRI, used as control, resulted in a painful response by the patient.

The average age of patients with positive discography was 40.7 years (range, 25-56 years) and for negative discography 43.1 years (range, $30-55$ years).

Men had a positive discography rate of $43.5 \%$ versus $40 \%$ in women.

\section{MRI findings associated with discography}

The following are the OR and diagnostic characteristics (and their 95\% CIs) evaluating the association between each MRI finding and positive discography. None of the associations were statistically significant:

- Protrusion disc (OR = 3.5; 95\% CI: 0.7-18); sensitivity $=42.9 \%$, specificity $=82.3 \%$; positive predictive value $[\mathrm{PPV}]=23.3 \%)$

- Hernia $(\mathrm{OR}=3.5 ; 95 \%$ CI: 0.4-47.6; sensitivity $=$ $21.4 \%$; specificity $=94.1 \%$; PPV= 30.4\%)

- HIZ (OR $=8.5 ; 95 \%$ CI: $0.8-91$; sensitivity $=28$ specificity $=94 ; \mathrm{PPV}=0.14$ )

- Modic change (OR $=6.4 ; 95 \%$ CI: 0.5-73; sensitivity $=21.4$ specificity $=94 ; \mathrm{PPV}=0.3)($ Table 2$)$.

MRI findings had very low sensitivities to identify patients with lumbar discogenic pain. The specificities of the findings: hernia, HIZ, and modic changes are high (Table $\mathbf{3}$ ).

\section{Clinical factors associated with discography}

- In the multivariate analysis, gender, age and involvement level were not significantly associated with positive discography.

- A history of surgery (OR $=3.3 ; 95 \%$ CI: $0.4-2.5)$, previous treatment ( $\mathrm{OR}=5.8 ; 95 \% \mathrm{CI}: 0.4-85)$, and having more than four episodes of LBP (OR $=3.8 ; 95 \% \mathrm{CI}$ : $.08-14)$ were associated with a greater odds of positive discography; however, these were not statistically significant.

VAS lumbar (OR adjusted $=0.6$; 95\% CI: 0.3-1.1); VAS leg (OR adjusted $=1 ; 95 \% \mathrm{CI}=0.8-1.2$ ) and Oswestry score (OR adjusted $=.9 ; 95 \%$ CI: $0.8-1$ ) were used to assess the 
pain and loss of function of patients with LBP. None of these variables were significantly associated with positive discography (Figs 3-7).

\section{Disc resistance}

The end point was evaluated as with or without resistance to the injection of dye into the disc. This study showed that patients with preserved disc resistance had a lower positivity test rate of $31.6 \%$, which was statistically significant. It raises a possible hypothesis that even with radiographic disc degeneration, some discs still maintain their structural properties not requiring surgery (Fig 8).

Injected volume in positive discography: $1.75 \mathrm{~mL}$ (1-3); negative discography: $2.18 \mathrm{~mL}(1.5-3)$;

Disc resistance-with resistance: positive discography 6 $(32 \%)$ and negative discography, $13(68 \%)$ and $P=.014$. Without resistance: positive discography, 7 (50\%) and negative discography, 7 (50\%) and $P>.05$ (Table 4).

\section{DISCUSSION}

There is growing evidence that the currently available techniques for the diagnosis of discogenic LBP are insufficiently accurate-this may explain some of the variability in outcomes of surgery for this important clinical problem. We have presented the results of 33 discographies of patients with chronic LBP, presumed to be discogenic in origin and compared them with MRI and clinical findings to elucidate data that could predict a positive discography that is highly sensitive to differentiate between symptomatic and asymptomatic disc degeneration, but it is an invasive procedure.

- With respect to clinical factors-gender, age, and involvement level were not significantly associated with positive discography. On the other hand findings such as protruding discs, herniated discs, HIZ and modic changes on MRI, demonstrated stronger associations; however, these were not statistically significant (ie, $95 \%$ CIs included 1.0) probably because of the small sample size.

- A history of spine surgery and having more than four episodes of LBP were also more strongly associated with positive discography; however, these results were also not statistically significant likely due to lack of study power.

- Patients whose primary complaint was LBP linked with sciatica had the highest incidence of positive discography than patients with LBP alone, but this was not statistically significant. Many patients had no imaging findings to justify sciatica. We believe that this is because MRI is a "static" test, held in the supine position, which cannot identify occult disc herniation or annular ruptures as discography can.

- VAS and Oswestry scales were not associated with positive discography.

- Carragee et al [2] published a study showing no statistical difference between patients having symptomatic and asymptomatic HIZ who underwent discography; However, there were questions of how a previously asymptomatic patient could relate 'reproducible' pain during the test, if they had never had pain before.
Fig 3 Relation between visual analogue scale (VAS) lumbar and discographic results.

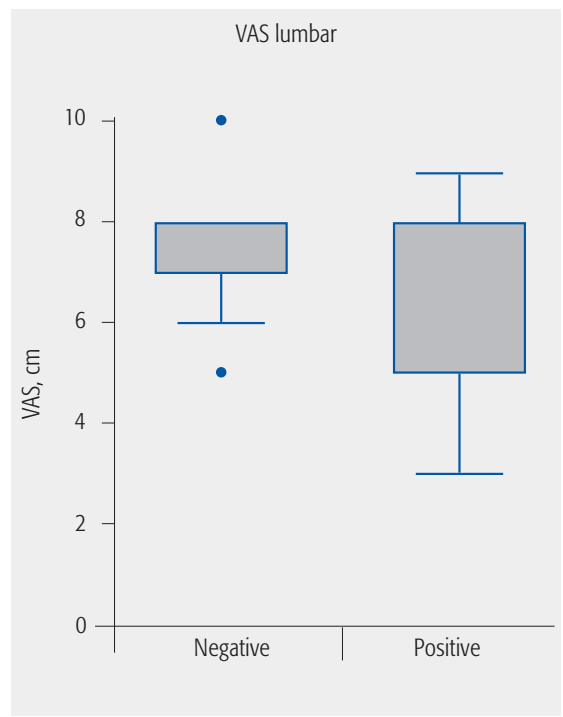

Fig 4 Relation between leg VAS with positive/negative discographic results.

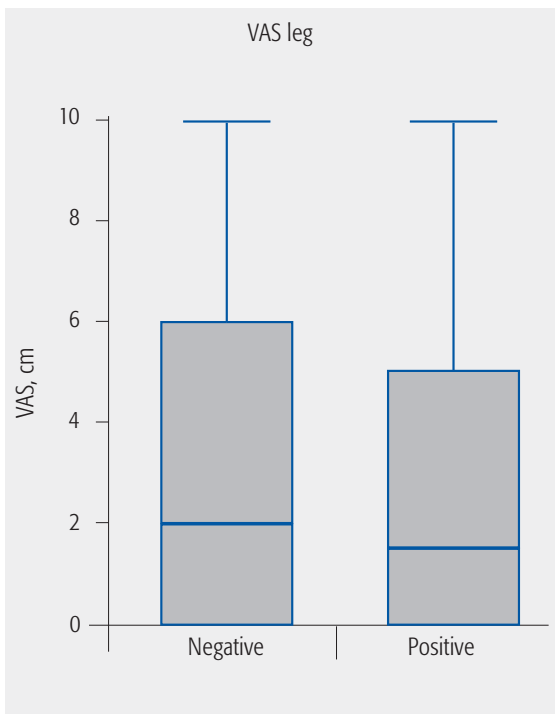

Fig 5 Relation between Oswestry test and discographic results.

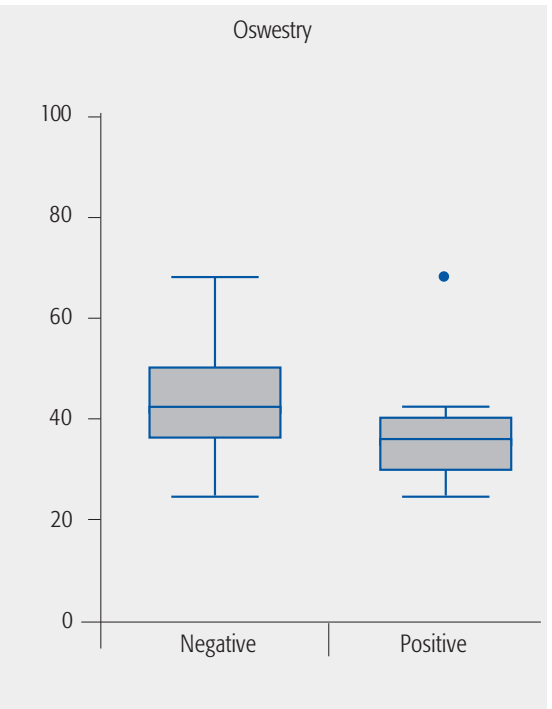


Schellhas et al [3] reported $87 \%$ agreement in patients with lumbar HIZ, which was similar to the present study.

- O'Neill and Kurgansky [4] showed in their study of pressure-controlled discography, a high incidence of true-positive when there was clinical correlation injecting the first few milliliters of contrast in the intervertebral disc. It is also important to rule out other causes of increased intradiscal pressure like posterior instrumentation (exclusion criterion). With all this in positive discography there is no need to inject more contrast in the disc that reproduces symptoms with small volumes of contrast. Meanwhile, in asymptomatic discs, it is important to inject a bit more contrast to confirm the test as a negative one.

- The end point was evaluated as with or without resistance to the injection of dye into the disc. This study showed that patients with preserved disc resistance had a lower test positivity rate of $31.7 \%$, which is statistically significant. It suggests that even with radiographic disc degeneration, some discs still maintain their structural properties not requiring surgery.

\section{Limitations}

The primary limitation of this study is the small sample size. Several potential associations demonstrated wide CIs. In addition, the study design was not the most appropriate for evaluating the use of discography in the management of chronic LBP. The ideal design for evaluating the utility of discography would be to compare outcomes in patients who did and did not receive a discography.

\section{CLINICAL RELEVANCE AND IMPACT}

At present, discography remains a controversial test, with clinicians either for or against it. Some disadvantages are that it is examiner dependent, requiring that the injection effectively penetrates into the intervertebral disc (which makes it an expert-dependent procedure). In addition, the patient must have the mental clarity and collaboration to report if the pain produced with the injection of contrast is the same as his/her primary complaint. Furthermore, it is a painful and invasive test, may induce further disc degeneration, and may risk neurological injury and/or infection along with inevitable radiation exposure to physician and patient. We determined that there was no definitive clinical or radiological (MRI) correlation with positive discography in our sample of patients.

\section{SUMMARY}

Discography may still be relevant as a diagnostic test before surgery in patients with DDD for diagnosis of discogenic back pain, since there is no definitive clinical or radiological correlation with a positive outcome in this specific group of patients.
Fig 6 Relation between injected volume and discographic results.

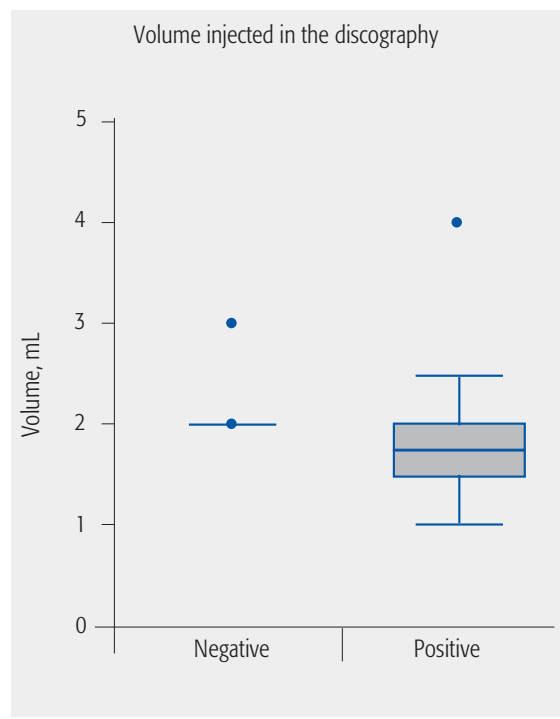

Fig 7 Relation between pain intensity and the result of the discography.

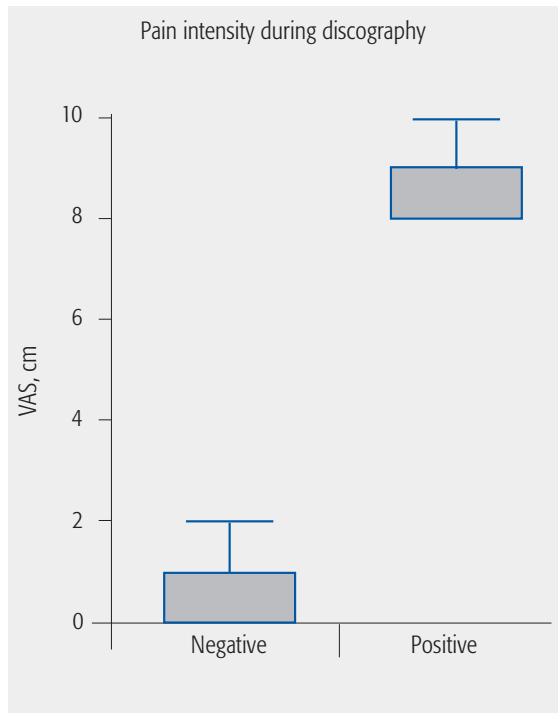

Fig 8 Relation between firm end point and positive discography.

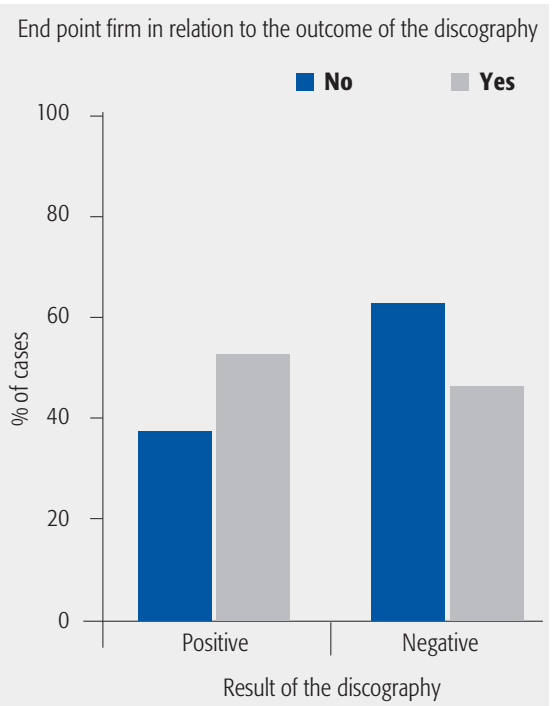




\section{REFERENCES}

1. Guyer RD, Ohnmeiss DD (1995) Lumbar discography: position statement from the North American Spine Society Diagnostic and Therapeutic Committee. Spine (Phila Pa 1976); 20(18):2048-2059.

2. Carragee EJ, Tanner CM, Yang B, et al (1999) False-positive findings on lumbar discography: reliability of subjective concordance assessment during provocative disc injection. Spine (Phila Pa 1976); 24(23):2542-2547.

3. Schellhas KP, Pollei SR, Gundry $C R$, et al (1996) Lumbar disc high-intensity zone: correlation of magnetic resonance imaging and discography. Spine (Phila Pa 1976); 21(1):79-86.

4. O'Neill C, Kurgansky M (2004) Subgroups of positive discs on discography. Spine (Phila Pa 1976); 29(19):2134-2139.

\section{EDITORIAL PERSPECTIVE}

Our reviewers raised concerns about this article because of the small size of the study population and data analysis not reaching statistical significance, thus not supporting its stated conclusions. After careful editorial review the decision to proceed with publication was made based on several valuable learning points regarding this controversial and provocative invasive testing method.

This article is a retrospective cohort study. The study design is not appropriate for clinical aim. The authors have no control arm, and hence there is no way to determine if a "positive discogram" is a false-positive. Lopez and colleagues assumed that positive discography in the setting of a patient with back pain confirms that the pain is discogenic in origin. How does one reconcile these results with those of Carragee et al [1], as cited by Lopez et al? In that study it was demonstrated that patients without any chronic low back pain (an appropriate control) had positive concordant pain responses with negative control discs. With respect to the secondary aim of Lopez and colleagues' study - they conclude that "... We determined that there was no definitive clinical or radiological (MRI) correlation with positive discography in our sample of patients." However, in the summary the author's state "Discography ... should continue on as a diagnostic test ... for diagnosis of back pain ... which leads to predict a positive outcome in a group of patients in particular." There were also more specific points raised:

The authors state that all discographies were performed between L3 and S1, and that one patient had three degenerative levels examined. For the patient with three-level discography, what was used as the control level?

Lopez et al note positive predictive values - they should have pointed out that positive predictive values are influenced by disease prevalence in the population, and as such the extrapolation of these results to MRI findings in a general population is limited.

Given the study design without a control and the results of the secondary aim (lack of definitive correlation), it is not clear to the reviewers of this study that the summary is supported by the data presented. Certainly, there is no substantiation by this article or others that lumbar discography can be used as a tool to search for sources of low back pain.

Lopez et al deserve credit for studying discography and hopefully will proceed with a prospective data-gathering approach and study outcomes of at least 2 years duration in both operatively and nonoperatively treated patients. Also, a greater appreciation of lumbar discs degeneration of discography control levels would be vital to the understanding of the physiological consequences of discography.

Carragee EJ, Tanner CM, Yang B, et al (1999) False-positive findings on lumbar discography: reliability of subjective concordance assessment during provocative disc injection. Spine (Phila Pa 1976); 24(23):2542-2547. 